\title{
Análisis multimedia del impacto del confinamiento por COVID-19 en menores con autismo
}

\section{Laura Manso-Refoyo ${ }^{1}$, Mㄹ Cristina González-Berrocal ${ }^{1}$, Sonia Verdugo-Castro ${ }^{2}$ y Mํㅡㄹ Cruz Sánchez-Gómez²}

Universidad de Salamanca, España | lauraman@usal.es; crisgonbe@usal.es | http://orcid.org/0000-0002-6893-9706; http://orcid.org/0000-0001-9254-773X

${ }^{2}$ Departamento de Didáctica, Organización y Métodos de Investigación, Facultad de Educación, Universidad de Salamanca, España | soniavercas@usal.es; mcsago@usal.es | http://orcid.org/0000-0002-9357-1747; http://orcid.org/0000-0003-4726-7143

Resumen: Introducción: La pandemia producida por el COVID-19 y los confinamientos domiciliarios que se han ordenado a causa de esta, han tenido impacto en los menores con Trastorno de Espectro Autista (TEA). Objetivo: Este trabajo se ha llevado a cabo para conocer el impacto social del COVID-19 en personas con autismo infantil, incluyendo la percepción y las reacciones de terceras personas. Como preguntas de investigación se plantean (1) ¿Cómo influye el confinamiento a los niños con autismo? (2) ¿Qué sugerencias educativas se pueden emplear para mantener una situación de estabilidad con niños con autismo, en situación de confinamiento? Método: Para la aplicación del estudio se ha seguido un proceso observacional de los entornos multimedia en Internet, profundizando en sitios web redes sociales, espacios audiovisuales e informativos. De tal manera que se ha optado por la metodología cualitativa, con corte fenomenológico. Para el análisis se han utilizado matrices de análisis y el CAQDAS webQDA, para la extracción de modelos visuales. Resultados: Del estudio se concluye que, desde la perspectiva social existe un desconocimiento por parte de la población sobre la incidencia de las medidas como el confinamiento en los menores con TEA. Este puede influir de manera diferente en cada menor dependiendo de sus características personales, familiares y sociales. Conclusiones: Por ello es necesario emplear intervenciones socioeducativas en niños con autismo.

Palabras clave: Trastorno del Espectro Autista; COVID-19; Confinamiento; Salud Mental; Conductas.

\section{Multimedia Analysis of the Impact of COVID-19 Confinement on Children with Autism}

Abstract: Introduction: The pandemic produced by COVID-19 and the home confinements that have been ordered because of it have impacted minors with Autism Spectrum Disorder (ASD). Objective: This work has been carried out in order to know the social impact of COVID19 on people with childhood autism, including the perception and reactions of third people. The research questions are (1) How does confinement influence children with autism? (2) What educational suggestions can be used to maintain a stable situation with children with autism in confinement? Method: For the study's application, an observational process of multimedia environments on the Internet has been followed, going deep into websites, socia networks, audiovisual and informative spaces. In such a way that we have opted for a qualitative methodology with a phenomenological approach. For the analysis, we used analysis matrices and CAQDAS webQDA to extract visual models. Results: The study concludes that, from a social perspective, there is a lack of knowledge on the part of the population about the incidence of measures such as confinement in children with ASD. This may influence each child differently depending on their personal, family and social characteristics. Conclusions: Therefore, it is necessary to use socio-educational interventions in children with autism.

Keywords: Autistic Spectrum Disorder; COVID-19; Confinement; Mental Health; Behaviors. 


\section{Introducción}

El autismo infantil es un trastorno del neurodesarrollo y se puede presentar durante el desarrollo del infante. Se caracteriza por su comienzo precoz, antes de los 3 años (Mardomingo, 1994), y abarca tres áreas fundamentales como la interacción social, los mecanismos de expresión y comunicación, y la presencia de conductas repetitivas y estereotipadas (Mardomingo, 1979). Este síndrome, fue explicado en 1943; se había visto como a un grupo de niños les resultaba imposible establecer vínculos habituales con personas y situaciones, además de una tendencia a la soledad autista, distanciándose de todo lo que se acercaba al niño (Kanner, 1971). Para conseguir una adquisición del lenguaje, el desarrollo de la comunicación e interacción social, y la compensación de deficiencias cognitivas, son importantes la escolarización en centros especializados y los programas educativos (Cohen \& Donellan, 1987). Estos programas pueden fomentar la mejora de conductas sociales inapropiadas, facilitando su adaptación al contexto.

Los datos del registro sociodemográfico "Autismo España", revelan que en la actualidad hay 2.116 personas con TEA (1.723 hombres y 392 mujeres) (Confederación Autismo España, 2019). Según investigaciones durante los últimos años se ha incrementado la prevalencia del Trastorno del Espectro Autista (TEA), alcanzando hasta el $2 \%$ de los niños (Fortea et al., 2013). Además, la reciente situación de pandemia producida por el COVID19 ha tenido varios efectos en personas con autismo.

En el presente trabajo se detallan dichos efectos como, por ejemplo, el confinamiento que ha provocado cambios de rutinas y conductas en niños autistas. Para prevenir estos cambios será esencial la figura del educador social y su intervención socioeducativa.

\section{Marco teórico}

De acuerdo con Rivero \& Rodríguez (2018) ha existido una evolución del concepto de autismo desde 1976 hasta 2018. También, Palomo (2012) investiga los síntomas de los Trastornos del Espectro Autista en los primeros dos años de vida. El estudio de Basso et al. (2017) revela que el diagnóstico del TEA no suele producirse antes de la edad escolar, lo que imposibilita acceder a programas de intervención tempranos y la orientación de los padres. Los resultados del estudio de Fortea et al. (2013) llevó a concluir que, en relación con las primeras sospechas, en el $79 \%$ de los casos fue la propia familia quien las tuvo, seguido de los profesionales de la educación (15\%) y sanidad (pediatras: $4 \%$ y psicólogos: $2 \%$ ). El $69 \%$ recibieron el primer diagnóstico durante los 3 primeros años, y el $32 \%$ antes de los dos años.

\subsection{Influencia del COVID-19}

Acerca de las repercusiones ocasionadas en niños con autismo, se debe mencionar la actual situación producida por la pandemia mundial, a causa del SARS-CoV-2 o COVID19 (Coronavirus Information for the COPD Community, 2020). Fruto de la pandemia, el Gobierno de España, declaró un Estado de Alarma el 14 de marzo de 2020 en todo su territorio durante 15 días, y sus sucesivas prórrogas, con medidas de restricción al movimiento de personas y la actividad económica (LP. ES \& EFE, 2020).

En personas con autismo, estos confinamientos domiciliarios pueden causar distintas respuestas emocionales y conductuales dependiendo de sus características personales, familiares y sociales. Para algunas personas con autismo la situación de aislamiento puede resultarles crítica y grave para su salud mental y física. Puede producirles alteraciones, como el aumento de conductas repetitivas, empeoramiento de su comportamiento o el desarrollo de la irritabilidad o la ansiedad (Hospital Sant Joan de Déu, 2020). También, Carmenate \& Rodríguez (2020) encontraron que durante la pandemia se han producido alteraciones como la agresividad, trastornos del sueño, irritabilidad, gritos y aparición de conductas estereotipadas. 
Ante estas situaciones de aislamiento, las personas con TEA tienen que hacer frente a dificultades comunicativas y carencia de lenguaje oral, siendo en ocasiones incapaces de expresar el dolor o malestar físico; por ello es fundamental que las personas con autismo tengan apoyos complementarios, para adaptarse a los cambios y dificultades que puedan agravarse durante este periodo (Horner et al., 2002). Por ello, el papel del cuidador es fundamental, y además es muy importante el autocuidado para mantener su estabilidad emocional (Hume et al., 2020). También, la figura del educador social es importante, dado que realiza intervención socioeducativa, por ejemplo, a través de tareas socioeducativas, con el objeto de seguir las rutinas que se tenían antes del confinamiento y para que su conducta no se vea afectada. Además, esta figura profesional puede orientar a la familia sobre qué pasos seguir (i Riera Romaní, 1998). Sobre el mantenimiento de rutinas en beneficio a la salud del niño, se recomienda promover el ejercicio físico, las actividades recreativas y de entretenimiento, acorde a la edad de la persona y espacio dónde se encuentre (Bellido, 2020).

En otra línea están los estudios como el de Romero et al. (2020), en el que se estudia la relación entre el estrés y el malestar psicológico de los padres y los problemas emocionales y conductuales en niños preescolares con TEA. Además de los profesionales, los progenitores o tutores legales del menor con autismo cumplen la función primordial de su cuidado, y también deben hacer frente a una serie de dificultades, tanto en el campo emocional como práctico (Cabezas, 2001). Según diversos estudios (Whitmore, 2016; Bonis \& Sawin, 2016) criar a un hijo con autismo es una experiencia que genera estrés y angustia, pudiendo ser mayor en los padres (Hayes \& Watson, 2013). Las investigaciones han demostrado que padres de hijos con TEA presentan alto riesgo de desarrollar depresión, en comparación con padres de hijos sin TEA (Bekhet \& Zauszniewski, 2013; Snow \& Donnelly, 2016; Van Steijn et al., 2016; Zablotsky et al., 2013).

Finalmente, Amorim et al. (2020) han estudiado el impacto del COVID-19 en niños con TEA. Los resultados muestran que tuvieron un impacto negativo en el manejo de las emociones frente a los niños del grupo de control. Los niños con autismo que no mantuvieron las rutinas estimaron niveles de ansiedad más altos, y sus padres también presentaron niveles más altos de ansiedad que los de control. Se concluyó que hay un fuerte impacto psicológico de la pandemia de Covid-19 de los niños con trastornos del neurodesarrollo y en sus cuidadores o familias.

\section{Metodología y método}

Se ha utilizado la metodología cualitativa, desde un corte fenomenológico (Martín Cilleros et al., 2020, Verdugo-Castro et al., 2019). Se persigue profundizar en la globalidad del contexto, desde los entornos digitales, para abordar desde las redes sociales y otros sitios web los cambios producidos en la sociedad, durante la pandemia, en relación con los niños que tienen TEA. Se ha seguido un proceso observacional de los entornos multimedia en Internet. A partir del estudio multimedia se ha realizado un análisis del contenido. Desde dichos espacios se pueden recoger sensaciones y sentimientos, como el miedo, vivencias y experiencias, como pérdida de rutinas o propuestas educativas y sociales, como la estimulación del lenguaje.

El objetivo del estudio es conocer el impacto social del COVID-19 en personas con autismo infantil, incluyendo la percepción y las reacciones de terceras personas. Como preguntas de investigación se plantean (1) ¿Cómo influye el confinamiento a los niños con autismo? (2) ¿Qué sugerencias educativas se pueden emplear para mantener una situación de estabilidad con niños con autismo, en situación de confinamiento?

El estudio se hizo a partir de un análisis multimedia de entornos de Internet (https://cutt.ly/JbEkQaG). Dado el auge que ha tenido el uso de las nuevas tecnologías durante la pandemia, se ha realizado un análisis de contenido de redes sociales, sitios web y entornos de comunicación. 
Para decidir qué elementos pasaban a la fase de análisis, se han lanzado diferentes búsquedas durante noviembre de 2020 en los motores de las redes sociales y de los navegadores con ecuaciones de términos como: "autismo AND COVID-19", "autismo AND confinamiento", "autismo infantil AND pandemia", y posteriormente, se ha realizado una lectura de los resultados, valorando la idoneidad para responder al objetivo y a las preguntas de investigación. Formaron parte del estudio un total de cinco blogs, dieciséis vídeos de YouTube, veintidós tweets, diecisiete noticias (del periódico digital El Mundo, Nueva España, revista Triodos, ABC, etc.) y, fueron revisadas algunas publicaciones de Instagram. La búsqueda fue realizada por dos autoras, y se centró en el espacio español, fundamentalmente. Posteriormente, la definición de categorías, la codificación, el análisis a través del CAQDAS webQDA y la interpretación de los resultados lo realizaron las cuatro autoras.

\subsection{Análisis de datos}

Tras el visionado del contenido audiovisual y la lectura del contenido, se ha generado un sistema categorial, mediante metacategorías, categorías y subcategorías (Martín Cilleros et al., 2020). El sistema de categorías se ha creado a partir de los términos clave más abordados en los resultados finalmente analizados. En el enlace https://cutt.ly/JbEkQaG se recoge el análisis de las fuentes finalmente seleccionadas. Tras el diseño del mapa conceptual, se ha realizado la reducción y transformación de los datos a partir del proceso de codificación. Este proceso ha permitido valorar las frecuencias de aparición de las diferentes categorías. Posteriormente, y ordenando la información en matrices, se ha procedido al análisis de contenido destacando las principales ideas, por tipología de fuente. Por último, con el soporte del software webQDA se han extraído modelos visuales como el árbol de códigos y la nube de palabras.

\section{Resultados}

En la Figura 1 se muestra el mapa conceptual diseñado a partir del estudio, mediante el CAQDAS webQDA.

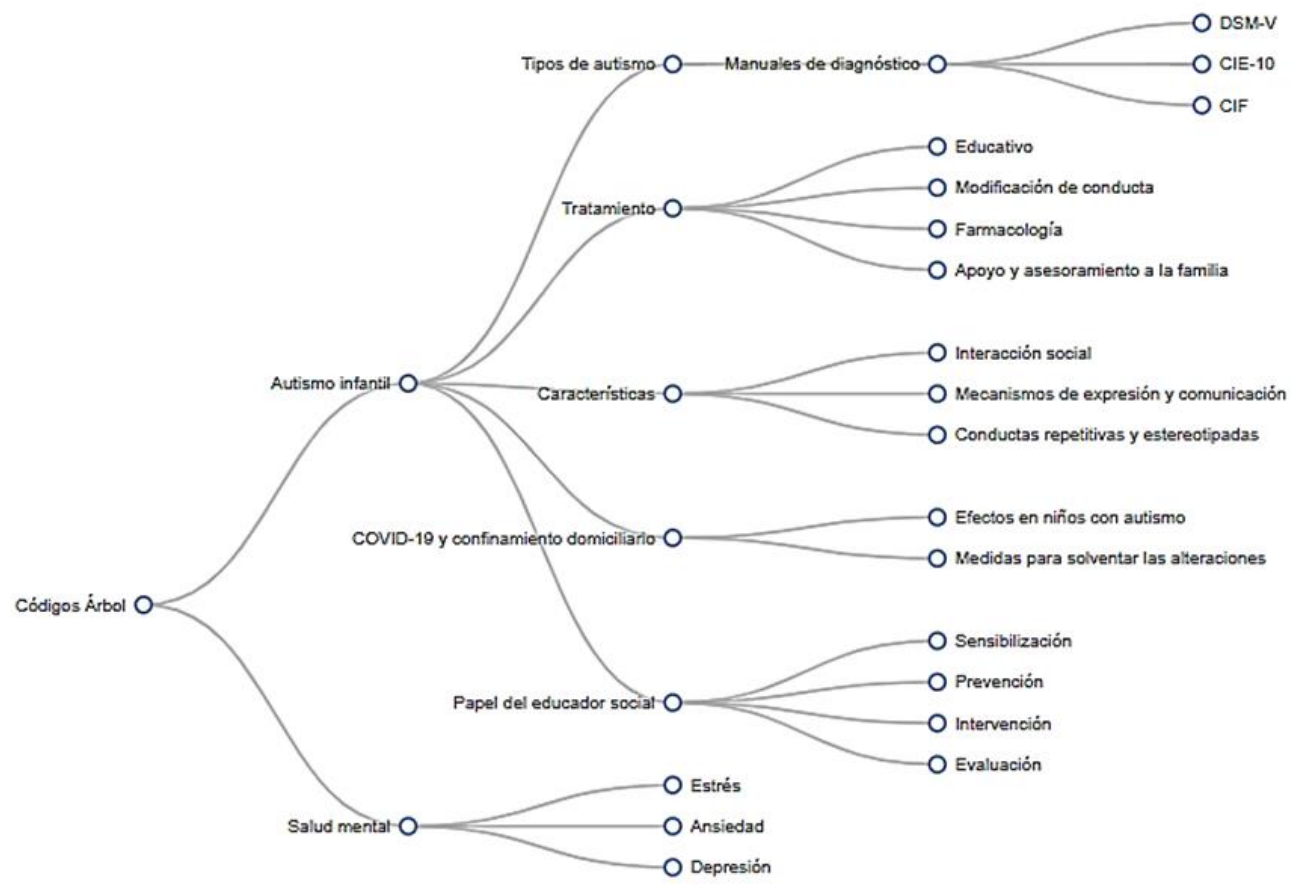

Fig. 1. Mapa conceptual. Fuente: Producción propia a partir de webQDA. 


\subsection{Resultados multimedia}

En la Figura 2 se presentan las frecuencias por categorías. En el enlace https://cutt.ly/JbEkQaG se recogen las categorías y el análisis desarrollado.

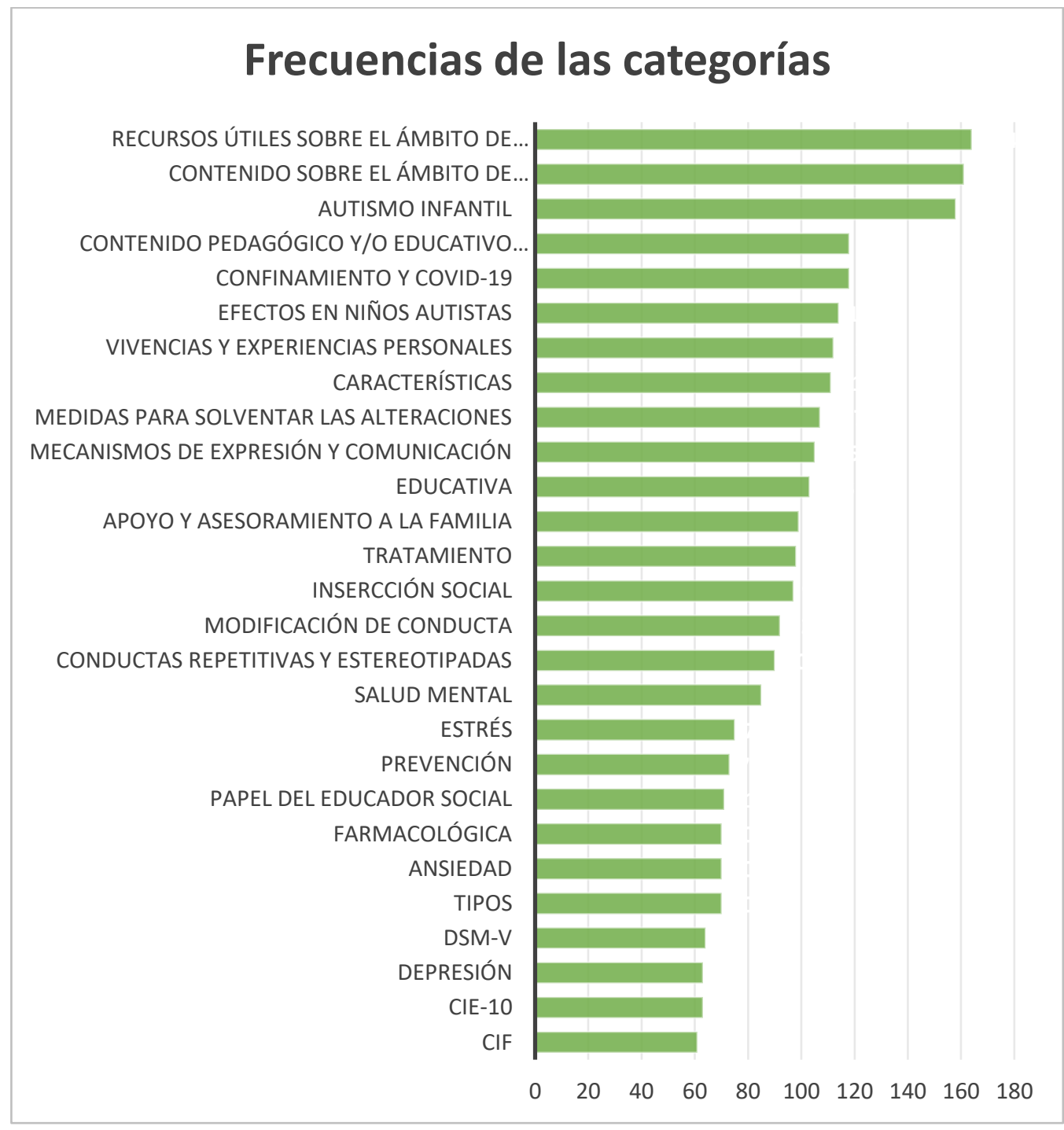

Fig. 2. Frecuencias de las categorías.

Fuente: Producción propia.

En uno de los blogs se habla del autismo como un trastorno neurobiológico del desarrollo, que afecta al funcionamiento cerebral y a la configuración del Sistema Nervioso, lo cual tiene como consecuencia dificultades en la comunicación e interacción social y también en la flexibilidad tanto del pensamiento, como de la conducta. Las manifestaciones clínicas de este trastorno pueden variar mucho en función del desarrollo personal, de los apoyos o de la presencia o ausencia de discapacidad intelectual asociada, así como también del nivel del desarrollo del lenguaje. Además, cabe destacar algunas unidades de análisis con mayor frecuencia. Por ejemplo, algunas de las recientes noticias revelan cómo afecta el confinamiento a los niños con autismo. El confinamiento condiciona a estos niños drásticamente, ya que no pueden seguir sus rutinas diarias, viéndose modificado su comportamiento y salud, perjudicialmente. Se suma otro problema y es que no han podido acudir a sus terapias habituales y los padres han tenido que crear nuevas rutinas en casa. Como culmen, cuando los niños salían a la calle en ocasiones han sido increpados por los vecinos desde los balcones. 
Por otro lado, durante la pandemia se han publicado vídeos dirigidos a niños con autismo. En uno de ellos (https://www.youtube.com/watch?v=19LVyNIOct0) se explican algunas pautas para sobrellevar el confinamiento con niños con TEA. Por ejemplo, explicarles con pictogramas o cuentos qué es el Coronavirus, enseñarles a lavarse las manos para prevenir el virus, intentar mantener las rutinas y ritmos de sueño y alimentación. También, en una noticia se explica qué significa el pañuelo azul que llevan algunos niños. Algunos padres de niños con autismo han relatado como han sido amenazados por sus vecinos por salir a la calle durante la emergencia sanitaria. Por ello, en redes sociales, ha surgido una iniciativa para identificar a estas personas, ya que necesitan salir frecuentemente a la calle, lo que no siempre es bien recibido. En esta línea, también aparecen noticias sobre el miedo de los niños con autismo y sus familias, sobre salir a la calle.

Además, en YouTube, se analizan dos categorías: COVID-19 y confinamiento, y autismo infantil. La categoría de autismo infantil tiene ligeramente mayor presencia, ya que se trata de un fenómeno reciente. También se presenta un vídeo externo a España (https://youtu.be/i1bMZFpcQ3E), sobre cómo en México el autismo no es tomado con seriedad por parte del sector público de salud, y la pandemia ha complicado esa situación. El autismo en México no es considerado una enfermedad, y aunque existe una ley general para la atención y protección a personas con la condición del espectro autista (Nueva Ley DOF, 2015), no se lleva siempre a cabo como vemos en el vídeo, donde las autoridades de salud no incluyen terapias y medicamentos en los programas de gratuidad. En otro vídeo se presenta a una madre relatando cómo se siente su hijo al no poder salir de casa, ir al colegio o a la terapia. Además, reclama la figura del educador para reducir el estrés de las familias.

Dentro de los blogs se encuentran muchas experiencias personales que han surgido durante la pandemia. Por ejemplo, una madre ofrece su visión del autismo: "M. tiene casi tres años y no habla. Sin embargo, es cariñoso y se comunica a su manera con su entorno. Pero algo no cuadra y un diagnóstico tardío despierta sensaciones abandonadas y heridas dormidas. El duelo tiene un principio y un fin y la lucha incansable por traer a Mario a nuestro mundo hace brotar una sonrisa eterna al leer cada línea de esta historia".

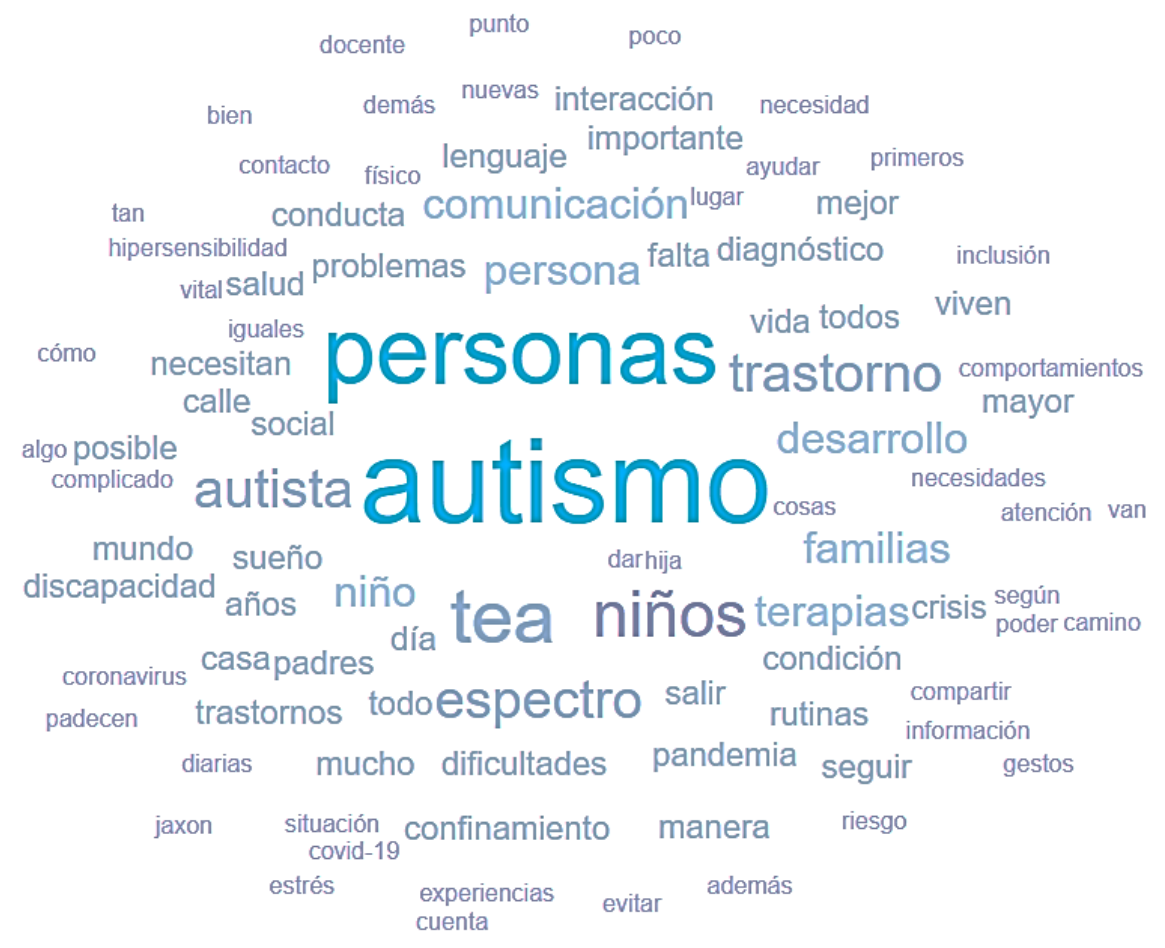

Fig. 3. Nube de palabras.

Fuente: Producción propia a partir de webQDA. 


\subsection{Resultados DAFO}

Las principales amenazas observadas son la falta de recursos, así como la falta de políticas inclusivas educativas. Por otro lado, se aprecia la necesidad de profesionales especializados. Otra de las grandes amenazas y más reciente ha sido el COVID-19 y el confinamiento, lo que ha desencadenado en estigmatizaciones, discriminaciones y violación de los derechos humanos. En cuanto a las oportunidades para los niños autistas, cabe destacar la creación de aulas específicas. También, destacar la importancia de la terapia conductual y los programas de capacitación para reducir las dificultades de comunicación y comportamiento social y aumentar la calidad de las personas con TEA y la de sus familias.

Como debilidades se identifica la dificultad para expresar empatía u otros sentimientos, así como entender los del resto. Se aprecian otras dificultades en la planificación de tareas a largo plazo o en la tarea de resumir y analizar la información importante de una conversación. Por último, decir que algunas de estas personas necesitan constante atención y apoyo en su vida. Sin embargo, también se encuentran varias fortalezas, cómo poder acceder a recursos especializados e individualizados. Cabe destacar a la familia como una de las redes de apoyo fundamental. Otro punto fuerte de estas personas es que suelen ser altamente competentes en un área particular y desarrollan ciertas habilidades muy por encima de la media; además, cuando se centran en su interés logran un gran éxito laboral. Por último, señalar la tendencia a la sinceridad.

Finalmente, en la Figura 3 se recoge una nube de palabras de los términos más frecuentes en los contenidos multimedia analizados.

\section{Discusión}

Dando respuesta a la segunda pregunta, se puede favorecer la estabilidad de niños con autismo en caso de confinamiento, a través de la ayuda a las familias, dotándolos de recursos gracias a la intervención de profesionales, planteando pautas, ya que estos niños han visto alteradas sus rutinas con las restricciones implantadas y han sufrido muchos cambios de conducta. Además, ya que los niños con autismo presentan alteraciones con el cambio de rutinas, hay que establecer otras nuevas a seguir, y desarrollar actividades que se llevaban a cabo en el centro escolar, aparte de fomentar el ejercicio físico en casa, el apoyo de la comprensión de la situación y la transmisión de sensación de control (Bellido, 2020). También tienen que hacer frente a dificultades comunicativas y a la falta de lenguaje oral (Horner et al., 2002). Igualmente, es esencial que las personas con autismo tengan apoyos complementarios para adaptarse a estos cambios, al igual que es importante su autocuidado para mantener su estabilidad emocional (Hume et al., 2020). Así pues, se ha detectado que algunos niños autistas no han podido ir a terapia, provocando que las familias se hayan adaptado para seguir rutinas.

El confinamiento en personas autistas puede causar distintas respuestas emocionales y conductuales dependiendo de sus características personales, familiares y de su entorno; como el aumento de conductas repetitivas, empeoramiento del comportamiento o el desarrollo de irritabilidad o ansiedad (Hospital Sant Joan de Déu, 2020). Asimismo, estas circunstancias acentúan los patrones de síndromes, trastornos y cuadros de discapacidad e incrementa el mal funcionamiento de las habilidades sociales (Horner et al., 2002).

\section{Conclusiones}

A través de la metodología cualitativa se estudia la realidad del autismo a través de las experiencias y opiniones personales.

Para dar respuesta al objetivo, desde la perspectiva social existe un desconocimiento por parte de la población al tratarse de personas con necesidades especiales, en este caso de niños con TEA. 
Como se ha analizado a través de las fuentes recopiladas (https://cutt.ly/JbEkQaG), las personas que increpaban o amenazaban a las familias que salían con los niños que lo necesitaban no mostraban respeto ni conciencia, lo que provocó grandes consecuencias y alteraciones en su salud. Por lo tanto, para que esto no suceda, es necesario emplear intervenciones socioeducativas. Los expertos del área educativa deben asesorar y emitir recomendaciones para las vivencias de duelo y miedo que muchos niños están viviendo, así como posibles espacios de "excepción" al confinamiento, con las medidas y limitaciones que se establezcan.

El confinamiento en niños autistas puede influir de manera diferente en cada uno dependiendo de sus características personales y familiares. Sin embargo, afecta de manera similar, ya que, dadas sus características, el confinamiento resulta ser perjudicial para su salud. El confinamiento domiciliario supone cambios emocionales y conductuales para los niños autistas, generando dificultades para adaptarse a esta situación. A través de la intervención socioeducativa del profesional de la educación social se debe perseguir promover la creación de redes de apoyo social que mejoren las habilidades personales y sociales de la infancia con Trastorno del Espectro Autista. Se puede usar una metodología de enseñanza-aprendizaje que fomente el aprendizaje colaborativo. Se trata de una manera de reducir la exclusión a la que se enfrentan algunos grupos más vulnerables y ante la cual se debe actuar y luchar por y para la inclusión.

Por último, destacar que esta investigación ha sido posible gracias a la metodología cualitativa, que ha posibilitado el análisis de contenido en blogs, noticias, vídeos, etc. Conocer la perspectiva y vivencias de las personas implicadas posibilita dibujar una imagen de la realidad abordada.

\section{Agradecimientos}

Este trabajo se ha realizado en el marco del Grado de Educación Social de la Universidad de Salamanca (España).

La investigación ha sido parcialmente apoyada por el Ministerio de Ciencia, Innovación y Universidades de España bajo un contrato predoctoral FPU (FPU017/01252).

\section{Referencias}

Amorim, R., Catarino, S., Miragaia, P., Ferreras, C., Viana, V. \& Guardiano, M. (2020). Impacto de la COVID-19 en niños con trastornos del espectro autista. Revista de Neurología, 71(8), 285291.

Basso, R., Backes, B. \& Alves, C. (2017). Diagnóstico del autismo: relación entre factores contextuales, familiares y del hijo. Revista de Psicología: Teoría y Práctica, 19(1), 152-163.

Bekhet, A. \& Zauszniewski, J. (2013). Evaluación psicométrica de la escala de cognición depresiva entre cuidadores de personas con trastorno del espectro autista. Archives of Psychiatric Nursing, 27(2), 96-100. https://doi.org/10.1016/j.apnu.2012.10.004

Bellido, P.J. (2020). Guía didáctica para personas con TEA como respuesta a las necesidades educativas devenidas por el confinamiento del Covid19. [Trabajo Fin de Grado]. Universidad Complutense de Madrid.

Bonis, S. \& Sawin, K. (2016). Riesgos y factores de protección para el autocontrol del estrés en padres de niños con trastorno del espectro autista: una revisión integrada de la literatura. Revista de enfermería pediátrica, 31(6), 567-579. https://doi.org/10.1016/j.pedn.2016.08.006

Cabezas, H. (2001). Los padres del niño con autismo. Revista Electrónica: Actualidades Investigativas en Educación. 1(2), 1-16.

Carmenate, I.D. \& Rodríguez, A. (2020). Repercusión psicológica en niños con trastorno del espectro autista durante el confinamiento por COVID-19. Revista Médica Multimed, 24(3), 690-707.

Cohen, D.J. \& Donnellan, A.M. (1987). Handbook of autism and pervasive developmental disorders. Nueva York, Wiley. 
Confederación Autismo España (2019). Se confirma el aumento de personas con autismo identificadas en España. Confederación Autismo España. Consultado el 23 de febrero de 2021, http://www.autismo.org.es/actualidad/articulo/se-confirma-el-aumento-de-personascon-autismo-identificadas-en-espana

Coronavirus Information for the COPD Community. (2020). COPD Foundation website. Consultado el 10 de diciembre de 2020, https://www.copdfoundation.org/Learn-More/l-am-a-PersonwithCOPD/Coronavirus-Information.aspx

Fortea, M. del S., Escandell, M.O. \& Castro, J.J. (2013). Aumento de la prevalencia de los trastornos del espectro autista: una revisión teórica. Revista Internacional de Psicología del Desarrollo y la Educación, 1(1), 747-764

Fortea, M. del S., Escandell, M.O. y Castro, J.J. (2013). Detección temprana del autismo: Profesionales implicados. Revista Española de Salud pública, 87(2), 191-199.

Hayes, S. \& Watson, S. (2013). El impacto del estrés parental: un metaanálisis de estudios que comparan la experiencia del estrés parental en padres de niños con y sin trastorno del espectro autista. Revista de autismo y trastornos del desarrollo, 43(3), 629-642. https://doi.org/10.1007/s10803-012-1604-y

Horner, R.H., Carr, E.G., Strain, P.S., Todd, A.W., \& Reed, H.K. (2002). Intervenciones de comportamiento problemático para niños pequeños con autismo: una síntesis de investigación. Revista de autismo y trastornos del desarrollo, 32(5), 423-446.

Hospital Sant Joan de Déu. (2020). Trastorno del espectro del autismo y coronavirus: información para familias. Consultado el 12 de diciembre de 2020 , https://www.sjdhospitalbarcelona.org/es/trastorno-espectro-autismocoronavirus-informacionfamilias

Hume, K., Waters, V., Sam, A., Steinbrenner, J., Perkins, Y., Dees, B., Tomaszewski, B., Rentschler, L., Szendrey, S., McIntyre, N., White, M., Nowell, S., \& Odom, S. (22 de mayo de 2020). Apoyar a las personas con autismo en tiempos de incertidumbre. AETAPI. http://aetapi.org/apoyo-apersonas-con-autismo-en-tiempos-de-incertidumbre/

I Riera Romaní, J. (1998). Concepto, formación y profesionalización de: El educador social, el trabajador social y el pedagogo social: un enfoque interdisciplinar e interprofesional. Nau Llibres. https://dialnet.unirioja.es/servlet/libro?codigo=209914

Kanner, L. (1971). Estudio de seguimiento de once niños autistas informado originalmente en 1943 J Autism Dev Disord 1, 119-145. https://doi.org/10.1007/BF01537953

Congreso de la Unión. (2015, 30 de abril). Ley General para la Atención y Protección a personas con la condición del espectro autista. Diario oficial de la Federación. http://www.diputados.gob.mx/LeyesBiblio/pdf/LGAPPCEA_270516.pdf

LP. ES \& EFE. (2020). Cronología del estado de alarma: 98 días de coronavirus que cambiaron la historia de España. Las provincias. https://www.lasprovincias.es/sociedad/cronologia-estadoalarma-20200620084732-nt.html

Mardomingo, M.J. (1979). Especificidad de los síntomas en el autismo infantil. En Autismo infantil: Cuestiones actuales (33-42). SEREM-APNA.

Mardomingo, M.J. (1994). Psiquiatría del niño y del adolescente. Díaz de Santos, S.A.

Martín Cilleros, M.V., Sánchez-Gómez, M.C., Verdugo-Castro, S., \& Verdugo Alonso, M.Á. (2020) Opiniones de la calidad de vida desde la perspectiva de la mujer con discapacidad. RISTI Revista Ibérica de Sistemas e Tecnologias de Informação, 38, 64-78. https://doi.org/10.17013/risti.38.64-78

Palomo, R. (2012). Los síntomas de los trastornos del espectro de autismo en los primeros dos años de vida: una revisión a partir de los estudios longitudinales prospectivos. Anales de Pediatría 76(1), 41-50.

Rivero, M. \& Rodríguez, J.M. (2018). Revisión histórica sobre el autismo. [Trabajo de fin de grado, Universidad de la Laguna].

Romero, M., Marín, E., Guzmán, J., Navas, P., Aguilar, J.M., Lara, P. \& Barbancho, M.A. (2020) Relación entre estrés y malestar psicológico de los padres y problemas emocionales y conductuales en niños preescolares con trastorno del espectro autista. Anales de pediatría. https://doi.org/10.1016/j.anpedi.2020.03.012 
Vol. 7 | Investigación Cualitativa en Educación: Avances y Desafíos

Snow, M. \& Donnelly, J. (2016). Factores que median los estados de ánimo disfóricos y ayudan a buscar el comportamiento entre padres australianos de niños con autismo. Revista de autismo y trastornos del desarrollo, 46(6), 1941-1952. https://doi.org/10.1007/s10803-016-2725-5

Van Steijn, D., Oerlemans, A., Van Aken, M., Buitelaar, J. \& Rommelse, N. (2016). La relación recíproca de TEA, TDAH, síntomas depresivos y estrés en padres de niños con TEA y / o TDAH. Revista de autismo y trastornos del desarrollo, 44(5), 1064-1076. https://doi.org/10.1007/s10803-013-1958-9

Verdugo-Castro, S. (2019). Detection of needs in the lines of work of third sector entities for unemployed women in situations of social exclusion. Pedagogía Social. Revista Interuniversitaria, O(34). https://doi.org/10.7179/PSRI_2019.34.12

Whitmore, K. (2016). Cuidado de relevo y estrés entre los cuidadores de niños con trastorno del espectro autista: una revisión integral. Revista de enfermería pediátrica, 31(6), 630- 652. https://doi.org/10.1016/j.pedn.2016.07.009

Zablotsky, B., Anderson, C. \& Law, P. (2013). La asociación entre la sintomatología del autismo infantil, la calidad de vida materna y el riesgo de depresión. Revista de autismo y trastornos del desarrollo, 43(8), 1946-1955. https://doi.org/10.1007/s10803-012-1745-z 